\title{
Substantiation of determination of strength per- formance of coarse-grained media by the oblique shear method
}

\author{
Sergey Shabaev ${ }^{1 *}$, and Sergey Ivanov ${ }^{1}$ \\ ${ }^{1}$ T.F. Gorbachev Kuzbass State Technical University, 28 street Vesennyaya, Kemerovo, the Russian \\ Federation
}

\begin{abstract}
The constantly increasing loads of vehicles predetermine the search for ways to improve both design methods, technological processes and the search for ways to improve the quality of road construction materials. The increasing volumes of open-pit coal mining require finding ways to improve the haul roads of open-pit coal mines. Thus, a qualitatively new approach is needed to determine the strength performance of coarse-grained media. In this work, it is proposed to determine the strength performance of granular media by the oblique shear method, since today there is no design method to determine the strength performance of coarse-grained media. The proposed design of the advanced oblique shear apparatus makes it possible to determine the strength performance of pre-compacted coarse-grained media with a particle size of up to $20-30 \mathrm{~mm}$. To avoid the effect of keyways and chaotic results, the optimum clearance between the upper and the lower holders should be 0.3 times the maximum particle size.
\end{abstract}

\section{Introduction}

One of the most important operations in open-pit mining is rock mass hauling, which is carried out, in the vast majority of cases, by dump trucks. To reduce the unit cost of mining, including at the expense of hauling costs, industrial enterprises tend to use dump trucks of especially large carrying capacity, up to 500 tons. This cannot but affect the state of open pit roads, which are built mainly from granular media: crushed rocks, crushed stone, crushed stone-sand and gravel-sand mixtures. To ensure the strength of haul road pavements, it is necessary to prevent deformations of these granular media caused by shear (tangential) stresses arising from loads from mining vehicles. And, despite the fact that to ensure the possibility and efficiency of the use of mining vehicles, haul roads with pavements including layers of asphalt concrete and geosynthetic materials are provided [1-3], granular media in the form of graded or ungraded crushed stone, including dry bound macadam, are the most used [4].

*Corresponding author: shabaev81@,rambler.ru 
At the moment, the strength performance of coarse-grained media have not been studied enough to be predictable or used as reference or normative data. This is due, among other things, to the fact that, despite a fairly large number of existing methods, including the direct shear, the triaxial compression and the field loading test according to the State Standard GOST 12248-2010, the rotational shear and the shear of ground pillars according to the State Standard GOST 20276-2012, as well as the oblique shear according to the IndustrySpecific Construction Standard VSN 29-76, only the last two methods can be used for coarse-grained media. It is also allowed to determine the strength of such media, both of disturbed and undisturbed structure, on a large-sized shear apparatus similar to a laboratory shear apparatus. However, if granular medium is used as a material for the construction of mine haul road pavements, then it must be compacted, which makes it possible to achieve a density exceeding the density under in-place conditions, which is simulated using these apparatuses. In this case, all known methods become inapplicable due to the fact that they do not provide the required degree of compaction during preparation for the experiment. It is known that the mechanical properties of soils depend on their physical parameters, including density (porosity, void ratio) [5-8, etc.].

\section{Materials and methods}

The shear apparatus, taking into account the required engineering modifications, is much easier to manufacture. When it is used, the reliability of the test results for coarse-grained media increases. However, to ensure its operation, a large number of holders, providing a different angle of inclination of the shear area, are required. Despite this drawback, it was decided to improve the shear apparatus in order to be able to determine the strength performance of coarse-grained media compacted with special equipment during the construction of layers of mine haul road pavements from them. During the manufacturing process, the following components were added:

- an additional load is provided, providing vertical pressure on the granular medium of $40 \mathrm{~g} / \mathrm{cm}^{2}$. This allows compacting the test medium either on a standard shaking table with an oscillation amplitude of $0.40 \pm 0.05 \mathrm{~mm}$ and an oscillation frequency of $48 \pm 3 \mathrm{~Hz}$, or on a hydraulic press under a pressure of $20 \mathrm{MPa}$, or any other to achieve the granular medium density (structure) corresponding to a density (structure) in natural conditions (at a construction site);

- an additional compacting device is provided, consisting of two hollow half-cylinders with stiffeners and connecting plates, which allows rigidly fixing the upper and lower holders relative to each other during compaction of the test medium in order to prevent their displacement;

- a bottom is additionally provided, which prevents the free flow of the test medium from the holders during their movement from a standard shaking or press table onto the base carriage under the axial pressure screw.

The advanced shear apparatus (Fig. 1) consists of two main units. The first unit is a frame with a rigidly fixed arm, which has an internal thread of the axial pressure screw. The second unit in the assembled state is a form in which the tested granular medium is directly placed when preparing and carrying out the test. 


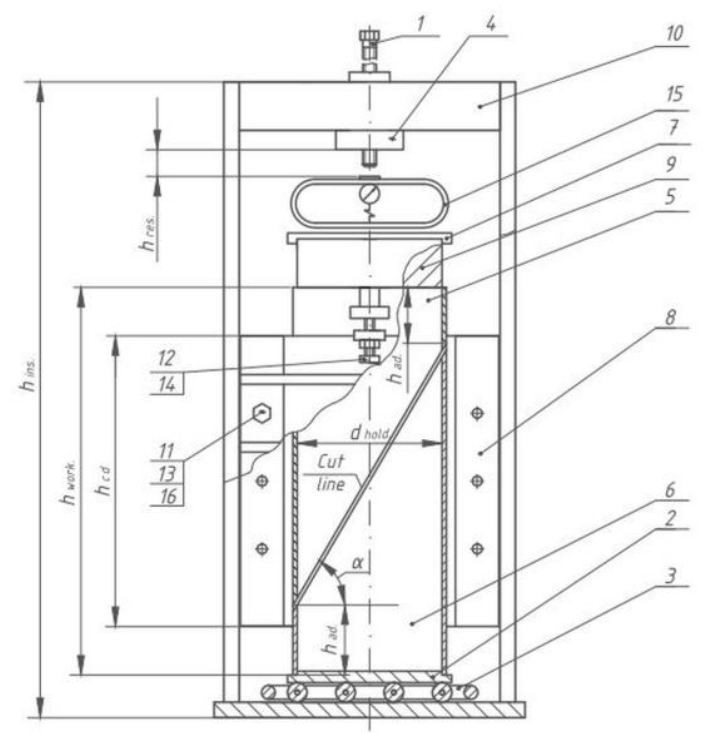

Fig. 1. Advanced oblique shear apparatus diagram

1 - axial pressure screw; 2 -bottom; 3 -base carriage; 4 -arm; 5 -upper holder; 6 -lower holder; 7 -adapter; 8 -half-cylinders of the compacting device; 9 -load; 10 -frame; 11, 13, 16-bolt connections; 12, 14 - adjusting screws; 15 - compression dynamometer

When preparing the test, the lower holder is installed on the bottom, two hollow halfcylinders with the stiffeners, which are fastened with the bolt connections without tightening and in the assembled state are a compacting device, are placed upon it. Then the upper holder is installed and the clearance is provided between the upper and the lower holders by adjusting the screws followed by tightening the bolt connections. After that, the test granular medium is poured into the assembled unit up to the upper face of the upper holder, the load providing a vertical pressure on the test medium of $40 \mathrm{~g} / \mathrm{cm}^{2}$ is placed on it and the unit is put onto a standard shaking table providing an oscillation amplitude of $0.40 \pm 0.05$ $\mathrm{mm}$ and an oscillation frequency of $48 \pm 3 \mathrm{~Hz}$, or onto a press plate. The granular material is compacted on a standard shaking table or on a press for 3 minutes, after which the unit is placed on the base carriage under the axial pressure screw. The half-cylinders of the compacting device are removed, the adapter is installed on the load and the compression dynamometer, the axial pressure screw is brought by rotation into contact with the latter.

When implementing the oblique shear method, the inner diameter of the holders $d_{\text {hold }}$ of the oblique shear apparatus is important, the values of which depend on the maximum size of the coarse-grained medium particles. Despite the fact that according to GOST 12248, the maximum particle size is limited to $1 / 5$ of the sample height, which is from $1 / 3$ to $1 / 2$ of the inner diameter, that is, the sample height is less than its diameter, when implementing the oblique shear method, the situation is the opposite - the sample diameter is less than its height. Taking into account the results of studies [10-12], we can say that in order to reduce the influence of the wall effect, the inner diameter of the holders should be at least 5-7 times greater than the maximum particle size.

The design diagram for the oblique shear test is shown in Fig. 2. From this diagram it is clear that the normal and shear stresses arising along the shear area are equal to: 


$$
\begin{gathered}
\sigma=\frac{4 \cdot F \cdot \cos ^{2} \alpha}{\pi \cdot d^{2}}, \\
\tau=\frac{4 \cdot F \cdot \sin \alpha \cdot \cos \alpha}{\pi \cdot d^{2}} .
\end{gathered}
$$

where $F$ - force resulting from external load; $\alpha$ - angle of inclination of the cut line (shear area) relative to the horizontal plane.

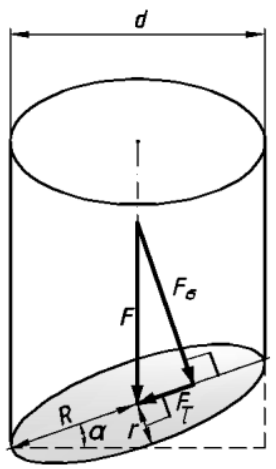

Fig. 2. The design diagram for the oblique shear test

It is clear that the total working height of the holders $h_{\text {work }}$ in the assembled state will depend both on the inner (outer) diameter of the holders and on the value of the angle of inclination of the shear area (the cut line angle) $\alpha$. It is known that the maximum angle of inclination of the shear area is $60^{\circ}$ [3], and therefore, taking into account the provision of an additional distance $h_{\text {ad }}$ equal to $30 \mathrm{~mm}$, it is possible to determine the total working height of the holders. Recommended values of inner diameter and total working height of the holders are given in Table 1

Table 1. Recommended values of inner diameter and total working height of the oblique shear apparatus holders

\begin{tabular}{|c|c|c|}
\hline $\begin{array}{c}\text { Maximum particle } \\
\text { size, mm }\end{array}$ & $\begin{array}{c}\text { Recommended inner diameter of } \\
\text { the holders, mm }\end{array}$ & $\begin{array}{c}\text { Recommended total working height of } \\
\text { the holders, mm, not less }\end{array}$ \\
\hline 10 & 70 & 200 \\
\hline 20 & 140 & 320 \\
\hline 40 & 280 & 560 \\
\hline 80 & 560 & 1050 \\
\hline
\end{tabular}

The data presented in Table 1 indicate that the total working height of the holders, with a maximum particle size of more than $20 \mathrm{~mm}$, can exceed the maximum permissible thickness of the compacted layer, which is, as a rule, no more than $30-50 \mathrm{~cm}$, even though the active zone can reach more than $3 \mathrm{~m}$ [13]. Therefore, in order to avoid the possibility of insufficient consolidation of the granular medium containing particles of more than $20 \mathrm{~mm}$ in size, it is advisable to fill the test medium into the assembled unit in two stages. At the first stage, approximately half of the design volume of the granular medium is filled with compaction under given conditions. At the second stage, the upper part of the already compacted granular medium is loosened to a depth corresponding to the maximum particle size, and the second half is filled with final compaction under the same compaction conditions.

It is also clear that the total mass of the unit, together with the granular medium containing particles with a size of more than $20 \mathrm{~mm}$, will be large enough to be able to move it only by human efforts, and therefore, in this case, it is necessary to provide devices for mechanizing these processes. In addition, not all presses and shaking tables are able to accommodate units 
of this size, and, therefore, when implementing the oblique shear method with preliminary compaction of the granular medium to a degree comparable to the degree of compaction achieved in field conditions, it is advisable to limit the maximum grain size to $20-30 \mathrm{~mm}$. If it is necessary to test granular media containing larger particles, it is advisable to place the entire apparatus directly on a press with a large base plate and ensure that the coarsegrained medium can be removed from the units after testing.

\section{Results}

In accordance with the well-known technique for a granular medium testing, it is necessary to conduct tests at least three different values of the angle of inclination of the shear area. The standard angles are: $30^{\circ}, 40^{\circ}, 50^{\circ}$, and $60^{\circ}$. However, if we analyze the dependences of the change in the arising shear stresses on the normal stresses acting on the shear area located at different angles $\alpha$ and compare them with the ultimate values of the shear resistance of granular media at different values of the angle of internal friction $\varphi$ (specific adhesion is conventional), then the following conclusions can be drawn (Fig. 3):

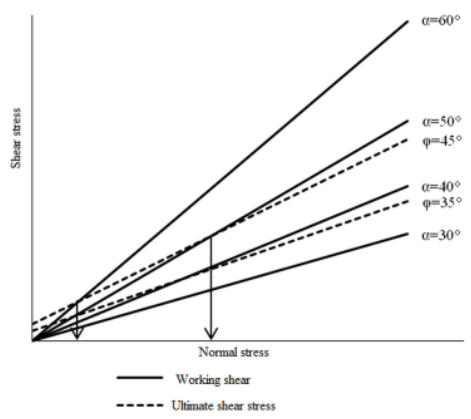

Fig. 3. Dependences of shear stresses on the magnitude of normal stresses arising along a shear area located at different angles $\alpha$ and ultimate granular media shear stresses with different angles of internal friction $\varphi$

1. Since in most granular media the angle of internal friction is in the range from 30 to $45^{\circ}$, and in the absence or small number of particles less than $1 \mathrm{~mm}$, as a rule, exceeds $40^{\circ}$, then at angles of inclination of the shear area $\alpha=30^{\circ}$ and $\alpha=40^{\circ}$, it is not recommended to test such media, since the line of growth of the ultimate shear stresses cannot cross the lines of the working shear stresses arising along the shear area from the external load. In this case, shear is possible only when the granular media is destroyed (crushed) to a state in which its angle of internal friction decreases to a value corresponding to the angle of inclination of the shear area.

2. If you test a granular medium at angles of inclination of the shear are $\alpha=50^{\circ}$ and $\alpha=60^{\circ}$, then two points will not be enough to reliably determine the desired strength performance. In this regard, it is necessary to conduct tests at intermediate angles $\alpha=45^{\circ}$ and $\alpha=55^{\circ}$.

3. When the value of the angle of internal friction of the granular media is significantly different from the angle of inclination of the shear area, the line of growth of the ultimate shear stresses intersects the line of the working shear stresses that arise along the shear area from the external load, at insignificant normal stresses. This can lead to the fact that the obtained point will not lie on the line described by Coulomb's law, since it is known that the initial section of the shear curve is non-linear for low values of the normal stress. 
Among other things, to determine the influence of the size of the clearance between the upper and lower holders of the advanced oblique shear apparatus with an inner holder diameter of $149 \mathrm{~mm}$ and a holder height of $400 \mathrm{~mm}$ on the keyway effect, a corresponding experiment was conducted. Despite the generally accepted requirements for the optimization parameter, in this case it was not only an unique probably significant numerical value, since, among other things, the senses were used in its determination. The results of the experiment are presented in Table 2 .

Table 2. Results of an experiment to assess the influence of the clearance between the upper and lower holders on the keyway effect

\begin{tabular}{|c|c|c|c|c|}
\hline $\begin{array}{c}\text { S } \\
\text { No. }\end{array}$ & $\begin{array}{l}\text { Cutting line } \\
\text { angle, degrees }\end{array}$ & $\begin{array}{l}\text { Clearance } \\
\text { size, } \mathrm{mm}\end{array}$ & $\begin{array}{l}\text { Breaking load, } \\
\mathrm{kN}\end{array}$ & Notes \\
\hline 1 & 2 & 3 & 4 & 5 \\
\hline & & Coarse me & with a particle & ze of 10 to $20 \mathrm{~mm}$ \\
\hline 1 & \multirow{13}{*}{50} & \multirow{5}{*}{8} & 1.86 & \\
\hline 2 & & & 8.04 & \\
\hline 3 & & & not determined & $\begin{array}{l}\text { Shift when removing the compacting } \\
\text { device }\end{array}$ \\
\hline 4 & & & 4.51 & \\
\hline 5 & & & not determined & Uneven clearance between the holders \\
\hline 6 & & \multirow{5}{*}{6} & 9.23 & \\
\hline 7 & & & 9.35 & \\
\hline 8 & & & 9.05 & \\
\hline 9 & & & 5.24 & Error \\
\hline 10 & & & 8.18 & \\
\hline 11 & & \multirow{3}{*}{4} & \multirow{3}{*}{ more than 15} & \multirow{3}{*}{$\begin{array}{l}\text { Sound waves characterizing the fracture of } \\
\text { particles }\end{array}$} \\
\hline 12 & & & & \\
\hline 13 & & & & \\
\hline \multicolumn{5}{|c|}{ Coarse media with a particle size of 5 to $10 \mathrm{~mm}$} \\
\hline 14 & \multirow{11}{*}{47.5} & \multirow{3}{*}{4} & not determined & Uneven clearance between the holders \\
\hline 15 & & & not determined & Uneven clearance between the holders \\
\hline 16 & & & 0.78 & \\
\hline 17 & & \multirow{5}{*}{3} & 7.48 & \\
\hline 18 & & & 8.98 & \\
\hline 19 & & & 8.42 & \\
\hline 20 & & & 7.82 & \\
\hline 21 & & & 8.00 & \\
\hline 22 & & \multirow{3}{*}{2} & more than 15 & \multirow{3}{*}{$\begin{array}{l}\text { Sound waves characterizing the fracture of } \\
\text { particles }\end{array}$} \\
\hline 23 & & & more than 15 & \\
\hline 24 & & & more than 15 & \\
\hline \multicolumn{5}{|c|}{ Coarse media with a particle size of 2.5 to $5 \mathrm{~mm}$} \\
\hline 25 & \multirow{10}{*}{45} & \multirow{4}{*}{2} & not determined & $\begin{array}{l}\text { Shift when removing the compacting } \\
\text { device }\end{array}$ \\
\hline 26 & & & 1.59 & \\
\hline 27 & & & not determined & $\begin{array}{c}\text { Shift when removing the compacting } \\
\text { device }\end{array}$ \\
\hline 28 & & & 0.46 & \\
\hline 29 & & \multirow{6}{*}{1.5} & 2.52 & \\
\hline 30 & & & not determined & Uneven clearance between the holders \\
\hline 31 & & & 2.49 & \\
\hline 32 & & & 2.67 & \\
\hline 33 & & & 2.31 & \\
\hline 34 & & & not determined & Shift when removing the compacting \\
\hline
\end{tabular}




\begin{tabular}{|c|c|c|c|c|}
\hline & & & device \\
\cline { 4 - 4 } \cline { 4 - 4 } & & 2.18 & \\
\cline { 3 - 4 } & & more than 15 & $\begin{array}{c}\text { Sound waves characterizing the fracture of } \\
\text { particles }\end{array}$ \\
\hline 36 & 1 & m & & \\
\hline
\end{tabular}

\section{Conclusions}

The experimental data also show that at an angle of inclination of the shear area $\alpha=50^{\circ}$, granular media consisting of particles smaller than $2 \mathrm{~mm}$ are displaced at a small normal stress, that is, there is a high probability that the obtained experimental data cannot be reliably described by the Coulomb's law.

1. The proposed advanced laboratory oblique shear apparatus design, which additionally provides load, compacting device and bottom, allows determining the strength performance of granular media containing particles of any size without limitation on their content. This is achieved due to the possibility of achieving the granular medium density (structure) corresponding to the density (structure) in a natural conditions (at a construction site) by compaction on a shaking table or a press.

2. It is proved that the standard values of the angle of inclination of the shear area of $30^{\circ}, 40^{\circ}, 50^{\circ}$ and $60^{\circ}$, implemented using the oblique shear test, are not suitable for determining the strength performance of granular media, consisting mainly of particles larger than $0.5 \mathrm{~mm}$.

3. Strength performance of coarse-grained media, compacted to a degree comparable to the degree of compaction achieved in field conditions, can be determined by the oblique shear method using an advanced portable oblique shear apparatus, if they contain particles up to $20-30 \mathrm{~mm}$ in size.

4. In order to avoid the keying effect and chaotic results, the optimal clearance between the upper and lower holders should be 0.3 of the maximum size of the coarse-grained medium particles.

5. To obtain the confidence interval of the measured value of the breaking load $\pm 10 \%$ of the weighted average value with a confidence probability of $90 \%$ when implementing the oblique shear method, taking into account possible errors, the test must be repeated with the same factors at least 5 times.

\section{References}

1. A.A. Khristoforova, M.D. Sokolova, S.E. Filippov, S.M. Semenova, I.N. Gogolev, Chemical Technologies and Nanomaterials: the materials of the international conference, 41 (2014)

2. A.A. Khristoforova, M.D. Sokolova, B.N. Zarovnyaev, A.N. Akishev, Mining Journal, 3, 47 (2016)

3. G.V. Solovyev, Procedia Engineering, 189, 666 (2017)

4. S.A. Arefiev, Natural and technical sciences, 1, 38 (2015)

5. B.A.Vallerga, H.B. Jeed, C.Z. Monismith, R.J. Coopes, Special technical publication, 212 (1957)

6. P.I. Gordienko, Proceedings of V.V. Kuibyshev Moscow Institute of Civil Engineering, 32 (1960) 
7. L.N. Rasskazov, Proceedings of Scientific Research Institute VODGEO, 19 (1968)

8. M.P. Lysenko, Composition and physical and mechanical properties of soils (Nedra, Moscow, 1980)

9. G.E. Mueller, Powder Technology, 203, 626 (2010)

10. M.M. Roozbahani, Powder Technology, 224, 46 (2012)

11. C.M. Wensrich, Powder Technology, 219, 118 (2012)

12. V.G. Bondarev, Modern trends in the development of science and technology, 2-1, 12 (2015) 